\title{
The promise of natural language processing in healthcare
}

Rohin Attrey, Alexander Levit

\section{ABSTRACT}

The healthcare industry generates data at a rapid rate, with no signs of slowing down. A large portion of this information takes the form of unstructured narrative text, making it difficult for computer systems to analyze the data in a usable format. However, automated analysis of this information could be incredibly useful in daily practice. This could be accomplished with natural language processing, an area of artificial intelligence and computational linguistics that is used to analyze and process large sets of unstructured data, namely spoken or written communication. Natural language processing has already been implemented in many sectors, and the industry is projected to be worth US\$16 billion by 2021. Natural language processing could take unstructured patient data and interpret meaning from the text, allowing that information to inform healthcare delivery. Natural language processing can also enable intelligent chatbots, interacting and providing medical support to patients. It has the potential to aid physicians by efficiently summarizing patient charts and predicting patient outcomes. In hospitals, it has the ability to analyze patient satisfaction and facilitate quality improvement. Despite current technical limitations, natural language processing is a rapidly developing technology that promises to improve the quality and efficiency of healthcare delivery.

\section{INTRODUCTION}

When electronic health records (EHR) were introduced, they promised a wide range of solutions for the healthcare system. EHRs would offer portability of large volumes of information, heightened security, and faster access to patient information. ${ }^{1-3}$ However, EHRs have also introduced new challenges that are affecting the clinicians and patients they sought to help. Although EHRs were intended to promote clinician efficiency, they have increased physician documentation time. ${ }^{4}$ Today, for every hour a physician spends with a patient, they spend 2 hours on EHRs and other administrative tasks. ${ }^{5}$ There is a clear need to improve the efficiency of EHR use, and other industries indicate that new data science technologies will be key to improving current EHR platforms.

Recent advances in data science are beginning to transform industries by providing insights from large data sets and improving outcomes while reducing costs. ${ }^{6}$ One such analytical approach is natural language processing (NLP). NLP is an area of artificial intelligence and computational linguistics that is used to analyze and process large sets of unstructured data, namely spoken or written communication. ${ }^{7}$ It is essentially a way in which a computer can extract meaning from written or spoken language. While rudimentary forms of NLP have existed since the 1950s, recent progress in statistics, processing speed, and artificial intelligence have made NLP more relevant for common implementation. NLP has been implemented with different types of infrastructures; there are more rudimentary forms such as retrieval-based models that have predefined responses, and more sophisticated generative models involving deep learning and distributed representations. ${ }^{8}$ As more sophisticated models become more widely available, NLP is expected to become more effective. For more information on the different structures that NLP can operate, see Young et al. ${ }^{8}$

Some examples of natural language processing include summarizing large texts, sentiment analysis, and automated question-answering applications such as the intelligent personal assistants that are found on most smartphones today (Apple Siri, Google Assistant, Microsoft Cortana). By 2021, the NLP industry is projected to be worth US\$16 billion globally. ${ }^{9}$ While NLP implementation in healthcare has been very limited, it has taken off in other industries and promises to deliver meaningful improvements in the healthcare industry.

\section{NLP FOR THE PATIENT}

Teletriage services, such as Telehealth Ontario, offer telephone access to triage nurses who can provide basic disease information and instructions for care. Unfortunately, this service is quite costly; in Ontario, the increasing cost of the program and trending decline in total call volume resulted in a Can $\$ 43$ cost per call in 2009.10 One innovative solution that could reduce the number of resources invested into teletriage is the use of NLP-enabled chatbots that provide spoken or written dialogue about a patient's health concerns. While we were not able to find any description of current NLP-enabled chatbots in healthcare settings, NLP tools have already been successfully implemented in analyzing clinical text, indicating that NLP-enabled chatbots could handle healthcare-related text. ${ }^{11-13}$ NLP-enabled chatbots have already been successfully implemented by companies such as Lufthansa, BMW, and Panasonic to deliver customer and technical support. ${ }^{14}$ While consumer support and healthcare provision have fundamental differences, important lessons for the development of NLP-enabled chatbots in healthcare can be gleaned from consumer industries.

A teletriage chatbot would free up the nurses and physicians who currently provide and monitor such services, who could otherwise apply their expertise in a face-to-face setting with patients. These chatbots are likely to cut costs, freeing up financial resources for other healthcare programs. Patients may even feel less hesitant communicating their concerns about health conditions that they find embarrassing or shameful, knowing that they are speaking in complete privacy with a machine incapable of passing social judgement, ultimately reducing barriers to care. Additionally, these chatbots could provide teletriage services in a patient's native 
language, creating an ease and comfort for them that current services do not provide. As NLP tools become more sophisticated, they will also be able to pick up on more subtle emotional and mental conditions. ${ }^{15}$ NLP-enabled chatbots could theoretically sense when a patient is in emotional distress and direct them to the appropriate resources. NLP can also be used to detect language patterns indicative of cognitive impairment, potentially aiding in the monitoring and diagnosis of cognitive decline and dementia. ${ }^{16,17}$

\section{NLP FOR THE PHYSICIAN}

Health professionals have been using computerized clinical decision support systems (CDSS) to aid in clinical decision-making for almost 50 years. $^{18}$ This includes EHRs that guide disease management, calculate drug dosing, or remind clinicians of patientspecific drug interactions and allergies. ${ }^{19}$ However, EHRs are loaded with unstructured text data - for this, NLP has the potential to take CDSS to the next level.

While EHRs do include structured patient data, a major portion of clinical observations are still in unstructured free-text form which would require sophisticated and reliable NLP algorithms for automated analysis. ${ }^{18}$ This includes all notes and reports that present in narrative form, including consultation reports, operative notes, and discharge summaries. In some cases, patient observations that might provide important inputs for CDSS can only be found within narrative data. NLP can therefore enable CDSS to monitor much more of the crucial data that can be found in EHRs. An exciting implementation of NLP-enabled CDSS has already demonstrated its potential for alerting clinicians to consider Kawasaki disease (KD) in emergency presentations. ${ }^{12}$ This NLP tool was trained on 22 emergency department summaries of patients who were later diagnosed with $\mathrm{KD}$, and when applied to 253 emergency department notes for children who were diagnosed with either KD or another febrile illness, the tool identified patients of high suspicion for KD with $93.6 \%$ sensitivity and $77.5 \%$ specificity. ${ }^{12}$ For rare conditions with serious complications, such as KD, automated NLP-enabled computer alerts about suspicious presentations could prove to be life-saving and cost-effective.

\section{NLP FOR THE RESEARCHER}

NLP also offers great methodological promise for qualitative research. Qualitative studies have become more common in medical literature, particularly for studying important social variables or subjective perspectives that are not conducive to quantitative investigation. ${ }^{20,21}$ A qualitative approach, such as a thematic analysis of patient interviews, can offer more data and unexpected insights than a quantitative approach such as a simple Likert scale-based survey. Indeed, in a study where physicians were asked to rate two fictitious abstracts with the same research topic and title, differing only by whether the design was quantitative or qualitative, primary care physicians appreciated the qualitative study as more clinically relevant. ${ }^{22}$ However, perceptions about time investment and subjectivity in data analysis still present barriers to pursuing qualitative studies or integrating qualitative approaches into mixed-methods studies. The time required to analyze qualitative data is more time consuming, as the data are inherently more unstructured. ${ }^{23}$ Though computer programs with lower levels of language processing have already been widely used in qualitative analysis, such as Atlas.ti, Hyper-research, and Nudist, these programs lack the sophistication that NLP could offer and require a lot of manual effort by researchers. ${ }^{24}$ Moreover, qualitative studies are often perceived to be less scientifically accurate, in part because they are perceived to involve more analytical subjectivity. ${ }^{22,25}$ These barriers could be overcome with the aid of NLP, which has already been shown to be a feasible approach to analyzing qualitative data. ${ }^{24}$ NLP can drastically reduce the time investment required for analyzing large collections of text data. ${ }^{24,25}$ Additionally, the higher level of reproducibility that can be achieved through analysis by a machine versus a potentially biased investigator should reduce the potential for analytical subjectivity. ${ }^{25}$ The development of NLP-enabled qualitative methods could therefore provide clinical investigators new tools for exploring research questions that may not have been pursued otherwise.

\section{NLP FOR HEALTHCARE MANAGEMENT}

Traditional customer satisfaction assessment tools have been limited to quantitative surveys. ${ }^{26}$ However, these surveys are unable to capture the full extent of the user experience and are subject to systematic biases. Questionnaires also miss a large portion of qualitative information that could otherwise be effectively used. NLP has the capability to take patient narratives in the form of unstructured and unrestricted feedback, written or spoken, and efficiently create meaningful summaries for management teams. This would provide new tools and insights that would help implement and monitor quality improvement initiatives.

\section{LIMITATIONS \& OTHER DIRECTIONS}

The legal and ethical concerns related to services enabled by artificial intelligence, such as job displacement, liability, and privacy, are highly relevant to evaluating the potential use of NLP in healthcare. These issues have been explored in greater detail elsewhere and are beyond the scope of this article. ${ }^{1,27,28}$ As long as NLP can improve healthcare quality and access, measures should be taken to implement NLP safely and ethically.

Although a number of studies show cases in which NLP was able to use qualitative information to predict an outcome, many current algorithms need to be further developed before being considered for implementation in CDSS. One recent study looked at suicidal ideation in a text-based analysis of mental health and produced a sensitivity and specificity of only $61 \%$ and $56 \%$ respectively. ${ }^{29}$ In developing NLP algorithms, there are also concerns regarding reinforcement of physician biases. The level of accuracy in predictive algorithms is contingent on the quality of data input. If these algorithms are using physician-generated data, and the collection processes of that data have inherent biases, the algorithm will reflect those underlying biases and assumptions. The development of NLP tools that rely on voice input will also rely 
on the accuracy of voice-to-text translation. Put simply, NLP with garbage input makes for garbage output.

Ultimately, NLP still promises to push the healthcare industry forward. As algorithms become more robust and more sophisticated, the widespread implementation of NLP will enable patients and healthcare professionals to tap into rich data sources, improving the quality and efficiency of healthcare delivery.

\section{REFERENCES}

1. Fernandez-Aleman JL, Senor IC, Lozoya PAO, et al. Security and privacy in electronic health records: A systematic literature review. J Biomed Inform. 2013 Jun 1;46(3):541-62. https://doi.org/10.1016/j. jbi.2012.12.003

2. Manca DP. Do electronic medical records improve quality of care? Yes. Can Fam physician Médecin Fam Can. 2015 Oct;61(10):846-7, 850-1.

3. Hoover R. Benefits of using an electronic health record. Nursing Critical Care. 2017 Jan;12(1):9-10. https://doi.org/10.1097/01. CCN.0000508631.93151.8d

4. Poissant L, Pereira J, Tamblyn R, et al. The impact of electronic health records on time efficiency of physicians and nurses: a systematic review. J Am Med Inform Assoc. 2005 Sep-Oct;12(5):505-16. https:// doi.org/10.1197/jamia.M1700

5. Sinsky C, Colligan L, Li L, et al. Allocation of physician time in ambulatory practice: a time and motion study in 4 specialties. Ann Intern Med. 2016 Dec 6;165(11):753. https://doi.org/10.7326/M16-0961

6. Raghupathi W, Raghupathi V. Big data analytics in healthcare: promise and potential. Heal Inf Sci Syst. 2014 Feb;2(1):3. https://doi.org/ 10.1186/2047-2501-2-3

7. Halper F. Advanced analytics: moving toward AI, machine learning, and natural language processing. [Internet] Best practices report. 2017. [cited 2018 Mar 16]. Available from: https://www.sas.com/content/ dam/SAS/en_us/doc/whitepaper2/tdwi-advanced-analytics-ai-mlnlp-109090.pdf.

8. Young T, Hazarika D, Poria S, et al. Recent trends in deep learning based natural language processing. IEEE Comput Intell M. 2018 July 20;13(3):55-75.

9. MarketsandMarkets. Natural Language Processing Market by Type (Rule-Based, Statistical, and Hybrid), Technologies (Recognition, IVR, OCR, Speech Recognition, Text Processing, Pattern \& Image Recognition), by Deployment Type, Vertical \& by Region - Global Forecast to 2021. [Internet] 2016. [cited 2018 Mar 16]. Available from: https://www.marketsandmarkets.com/Market-Reports/naturallanguage-processing-nlp-825.html.

10. Ontario Ministry of Health and Long-Term Care. [Internet] Teletriage health services. Vol. 13, Annual Report. 2011.

11. Johanna Johnsi Rani G, Gladis D, Manipadam MT, et al. Breast cancer staging using Natural Language Processing. In: 2015 International Conference on Advances in Computing, Communications and Informatics (ICACCI). IEEE; 2015 Aug 10-13. p. 1552-8. https://doi. org/10.1109/ICACCI.2015.7275834

12. Doan S, Maehara CK, Chaparro JD, et al. Building a natural language processing tool to identify patients with high clinical suspicion for Kawasaki disease from emergency department notes. Acad Emerg Med. 2016 May;23(5):628-36. https://doi.org/10.1111/acem.12925

13. Jackson RG, Patel R, Jayatilleke N, et al. Natural language processing to extract symptoms of severe mental illness from clinical text: the Clinical Record Interactive Search Comprehensive Data Extraction (CRIS-CODE) project. BMJ Open. 2017 Jan 17;7(1):e012012. https:// doi.org/10.1136/bmjopen-2016-012012
14. Coresight Research. 10 emerging startups in natural language processing (NLP) [Internet]. 2018. [cited 2018 Mar 16]. Available from: https://www.fungglobalretailtech.com/research/10-emergingstartups-natural-language-processing-nlp/.

15. Hirschberg J, Manning CD. Advances in natural language processing. Science. 2015 Jul 17;349(6245):261-6. https://doi.org/10.1126/science. aaa8685

16. Bynum W, Carrillo M, Davis R, et al. Cost-effective early detection of cognitive decline. [Internet] 2017 Dec 21. [cited 2018 Mar 16]. Available from: https://www.nia.nih.gov/sites/default/files/2018-01/finalcognitive-decline-summary.pdf

17. Roark B, Mitchell M, Hosom J-P, et al. Spoken language derived measures for detecting mild cognitive impairment. IEEE Trans Audio Speech Lang Processing. 2011 Sep 1;19(7):2081-90. https://doi. org/10.1109/TASL.2011.2112351

18. Demner-Fushman D, Chapman WW, McDonald CJ. What can natural language processing do for clinical decision support? J Biomed Inform. 2009 Oct;42(5):760-72. https://doi.org/10.1016/j.jbi.2009.08.007

19. Garg AX, Adhikari NKJ, McDonald H, et al. Effects of computerized clinical decision support systems on practitioner performance and patient outcomes. JAMA. 2005 Mar 9;293(10):1223. https://doi. org/10.1001/jama.293.10.1223

20. Poses RM, Isen AM. Qualitative research in medicine and health care: questions and controversy. J Gen Intern Med. 1998 Jan;13(1):32-8. https://doi.org/10.1046/j.1525-1497.1998.00005.x

21. Al-Busaidi ZQ. Qualitative research and its uses in health care. Sultan Qaboos Univ Med J. 2008 Mar;8(1):11-9.

22. Johansson EE, Risberg G, Hamberg K. Is qualitative research scientific, or merely relevant? Scand J Prim Health Care. 2003 Jan $12 ; 21(1): 10-4$.

23. Bryman A. Barriers to Integrating Quantitative and Qualitative Research. J Mix Methods Res. 2007 Jan;1(1):8-22. https://doi. org $/ 10.1177 / 2345678906290531$

24. Crowston K, Allen EE, Heckman R. Using natural language processing technology for qualitative data analysis. Int J Soc Res Methodol. 2012 Nov;15(6):523-43.

25. Tierney PJ. A qualitative analysis framework using natural language processing and graph theory. Int Rev Res Open Distrib Learn Distrib Learn. 2012 Dec;13(5):173-89. http://dx.doi.org/10.19173/irrodl. v13i5.1240

26. Lui JK, Laws SM, Li QX, et al. Plasma amyloid-beta as a biomarker in Alzheimer's disease: the AIBL study of aging. J Alzheimers Dis. 2010 June;20(4):1233-42. https://doi.org/10.3233/JAD-2010-090249

27. Lin P, Abney K, Bekey GA. Robot ethics: the ethical and social implications of robotics. Cambridge: MIT Press; 2012. 386 p.

28. Frankish K, Ramsey WL. The Cambridge handbook of artificial intelligence. Cambridge University Press; 2014 Aug. 354 p.

29. Cook BL, Progovac AM, Chen P, et al. Novel use of natural language processing (NLP) to predict suicidal ideation and psychiatric symptoms in a text-based mental health intervention in Madrid. Comput Math Methods Med. 2016 Aug;2016:8708434.http://dx.doi. org/10.1155/2016/8708434 